\title{
Remarks on Functional Integral Representations of Quantum Evolutions for Systems with Electromagnetic Fields
}

\author{
By
}

Lech S. PAPIEŻ

\begin{abstract}
The solutions of Schrödinger equation in the form of the Dyson series are used as the starting point for functional integral representations of wave functions. Quantum systems in the presence of the external electromagnetic fields are discussed in this respect. The results on measure theoretic approach to functional integral representations of quantum states are proved and related to the appropriate mathematical formulations of Feynman path integrals.
\end{abstract}

\section{$\S$ I. Introduction}

In this note we obtain the functional integral representations of solutions of Schrödinger equations describing systems with external electromagnetic fields and discuss their relations with Feynman paths integrals. The scalar and vector potentials used in these considerations are given by Fourier transforms of scalar and vector valued complex measures. To find the solution of Schrödinger equation in the form which allows the construction of its functional integral representation we use the method of Dyson series and allow the mass parameter $m$ to be any complex number in the upper half-plane, $m \in C_{+}=\{m: \operatorname{Im} m \geqq 0, m \neq 0\}$. Directly related to the Dyson series solutions are Feynman path integrals in the interpretation of Maslov-Čebotariev [MC], [CM], [CHRS] and Albeverio-HøeghKrohn $[\mathrm{AHK}]$. They give the meaning to $\int \exp \left[i / \hbar\left\{S_{0}(\omega)+S_{I}(\omega)\right\}\right] d \omega$ through $\int \exp \left[(i / \hbar) S_{0}(\omega)\right] d \mu_{I}(\omega)$, where $\mu_{I}$ is the measure on the space of paths constructed from the interaction part $\exp \left[(i / \hbar) S_{I}(\omega)\right]$ of the amplitude of probability $\exp \left[i / \hbar\left\{S_{0}(\omega)+S_{I}(\omega)\right\}\right]$. We give the explicit construction of measure $\mu_{I}$ on the space of absolutely continuous trajectories and prove that it is concentrated on the set of polygonal paths.

Communicated by H. Araki, June 29, 1988. Revised September 12, 1988. Department of Physics, University of Manitoba, Winnipeg, Canada R3T 2N2. 
We restrict our considerations to potentials which are time independent. All results can be generalized to time dependent scalar and vector fields [OPC]. These generalizations do not carry any conceptual novelites, and are technically troublesome, so we do not distract our attention to discuss them.

Let $x$ and $y$ denote points in $\mathbb{R}^{d}$. Then $x \cdot y$ is a scalar product of $x$ and $y,|x|$ is the Euclidean norm of $x$ and $x^{2}$ is the short notation for $x \cdot x$. We shall treat the quantum system described by the Lagrangian

$$
L(x, \dot{x})=\frac{m}{2} \dot{x}^{2}+a(x) \cdot \dot{x}-c \mathcal{V}(x)
$$

where $\mathcal{V}(x)$ and $a(x)$ denote scalar and vector (electromagnetic) fields, respectively. Thus the Hamiltonian of the investigated quantum system has the form

$$
\begin{aligned}
H\left(x, \nabla_{x}\right) & =\frac{1}{2 m}\left(\frac{1}{i} \nabla_{x}-a(x)\right)^{2}+\mathcal{V}(x)=-\frac{1}{2 m} \Delta_{x}+\overline{\mathcal{V}}\left(x, \nabla_{x}\right) \\
& =-\frac{1}{2 m} \Delta_{x}+\frac{i}{m} a(x) \cdot \nabla_{x}+\frac{i}{2 m} \nabla_{x} \cdot a(x)+\frac{1}{2 m} a^{2}(x)+\varnothing \nu(x)
\end{aligned}
$$

as we treat the Planck's constant $\hbar$ to be equal to 1 . The functions $a$ and $\mathscr{V}$ satisfy the following assumptions:

I. $\mathscr{V}: \mathbb{R}^{d} \rightarrow \mathbb{R}$ and at any $x \in \mathbb{R}^{d}$ the value of $\mathscr{V}$ is given by integral

$$
\checkmark(x)=\int_{R^{d}} \exp [i \alpha \cdot x] d \mu_{v}(\alpha)
$$

where $\mu_{v}$ is a compactly supported, scalar complex measure on $\mathbb{R}^{d}$ with a reflection property [OPC]. Let supp $\mu_{v} \subset S_{k}$ where $S_{k}$ is the ball of radius $k$ in $R^{d}$.

II. $a: \mathbb{R}^{d} \rightarrow \mathbb{R}^{d}$ and at any $x \in \boldsymbol{R}^{d}$ the value of $a$ is given by integral

$$
a(x)=\int_{R^{d}} \exp [i \alpha \cdot x] d \gamma(\alpha)
$$

where $\boldsymbol{\gamma}$ is a compactly supported, $\boldsymbol{C}^{d}$ valued measure on $\mathbb{R}^{d}$ with a reflection property [OPC]. Let also the condition supp $\gamma \subset S_{k / 2}$ be satisfied for measure $\gamma$.

We denote the class of potentials I by $\nu_{k}^{1}$ and, consequently, the class of potentials II by $\nu_{k / 2}^{d}$. For convenience, we shall denote by $\|\gamma\|$ the total variation of measure $\gamma(\|\gamma\|<\infty)$ and write $\mu^{\prime}=(1 / 2 m) \gamma * \gamma+\mu_{v}\left(\mu^{\prime} \in \nu_{k}^{1}\right)$ where $*$ denotes the convolution of measures $\gamma \in \nu_{k / 2}^{d}$. The fact that $\mu_{v} \in \nu_{k}^{1}$ and $\gamma \in \nu_{k / 2}^{d}$ implies that $\mathcal{V}$ and $a$ have bounded derivatives up to any order.

In all the foregoing considerations the condition of compact support for measure $\mu_{0}$ may be relaxed when the case without the electromagnetic field, i. e., $a \equiv 0$, is treated. We write then $\mu_{v} \in \nu^{1}$.

Definition 1. The $\mathcal{L}^{2}\left(\boldsymbol{R}^{d}\right)$ solutions of the Cauchy problem for Schrödinger equations determined by Hamiltonians of the form (1.2) are called Schrödinger evolutions and are denoted by $\operatorname{SEv}\left(\mathcal{A}_{1} ; \mathcal{A}_{2} ; \mathcal{A}_{3}\right)$ where $\mathcal{A}_{1}$ stands for the class of 
adnissible initial condiiions and $\mathcal{A}_{2}$ and $\mathcal{A}_{3}$ denote the fanilies to which potentials c) and a belong, respectively.

Throughout we put $h=1$; assume, if not stated otherwise, that $m \in \mathbb{C}_{+}$and take time parameter to belong to $\boldsymbol{R}^{+}=(0, \infty)$. In some circumstances $t$ will be restricted to interval $\left(0, T_{\gamma}\right)$ where $T_{\gamma}=|m|(2 e k\|\gamma\|)^{-1}$, $e$ being the base of the natural logarithm. In particular, let us notice that $m \in \boldsymbol{C}_{+}$allows $m$ to be specified as a real, positive number, and thus all results valid for $m \in C_{+}$are also true for the standard quantum mechanics.

It is known [OPC] that $\operatorname{SEv}\left(\mathcal{L}^{2}\left(\boldsymbol{R}^{d}\right) ; \nu_{k}^{1} ; \nu_{k / 2}^{d}\right)$ exists and is unique if initial condition $\psi_{0}$ and potentials $C V$ and $a$ are unambigously specified as elements of $\mathcal{L}^{2}\left(\boldsymbol{R}^{d}\right), \nu_{k}^{1}$ and $\nu_{k / 2}^{d}$, respectively. We write then $\phi(t ; m)=U(t ; m) \psi_{0} \in \operatorname{SEV}\left(\mathcal{L}^{2}\left(\boldsymbol{R}^{d}\right)\right.$; $\left.\nu_{k}^{1} ; \nu_{k / 2}^{d}\right)$ where $U(t ; m)$ is a family of bounded operators on $\mathcal{L}^{2}\left(\boldsymbol{R}^{d}\right)$. This family constitutes a semigroup with respect to parameter $t$, i.e.,

$$
U(t ; m) \bigcup(s ; m)=U(t+s ; m), \quad t \geqq 0, s \geqq 0, m \in C_{+}
$$

and is called the Schrödinger semigroup.

\section{$\S$ II. The Functional Representation of Wave Function from the Measure on the Space of Trajectories}

We start with the construction of the functional measure in the case of a system without an electromagnetic field (i.e., when $a \equiv 0$ ). The main steps are the same for the situation where an electromagnetic field is present (i.e., when $a \neq 0)$ and they will be presented in the second part of this section.

\section{Step 1. The Space of Trajectories}

Take $\widetilde{\mathscr{H}}=\mathcal{L}^{2}\left([0, t], \boldsymbol{R}^{d}\right)$, i. e., a space of measurable, square integral functions $\beta:[0, t] \rightarrow \boldsymbol{R}^{d}$. It is a separable Hilbert space. Let us denote by $\tilde{S}$ the set of step functions from $[0, t]$ into $\boldsymbol{R}^{d}$. $\tilde{S}$ is a dense subset of $\widetilde{\mathscr{H}}$. Any element $\tilde{s} \in \tilde{S}$ can be written as

$$
\tilde{s}(\tau)=\sum_{i=0}^{n} \alpha_{\imath} \cdot g_{t_{\imath}}(\tau), \quad \tau \in[0, t]
$$

where $n \in N=\{0,1,2, \cdots\}$ - the set of natural numbers; for fixed $n, t_{2}$ satisfy $0=t_{0}<t_{1}<\cdots<t_{n}<t$ and $\alpha_{\imath} \in \boldsymbol{R}^{d}, i=0,1, \cdots, n$; and function $g_{t_{\imath}}(\tau)$ is defined as

$$
g_{t_{i}}(\tau)=\left\{\begin{array}{rll}
0 & \text { if } & \tau<t_{i} \\
-1 & \text { if } & \tau \geqq t_{2} .
\end{array}\right.
$$

Every element $\beta$ from $\widetilde{\mathscr{T}}$ determines through the integral mapping

$$
\beta(\cdot) \longrightarrow \omega_{\beta}^{x}(\cdot)=-\int_{\cdot}^{t} \beta(\tau) d \tau+x
$$


the $\boldsymbol{R}^{d}$ valued, absolutely continuous function $\omega_{\beta}^{x}$ on $[0, t]$ which satisfies

$$
\omega_{\beta}^{x}(t)=x .
$$

In the set $\mathscr{H}^{x}$, which consists of elements $\omega_{\beta}^{x}$, we introduce a linear structure isomorphic to the space $\widetilde{\mathcal{H}}$ (i. e., $a_{1} \omega_{\beta_{1}}^{x} \oplus a_{2} \omega_{\beta_{2}}^{x}=\omega_{a_{1} \beta+a_{2} \beta_{2}}^{x} ; a_{1}, a_{2} \in \boldsymbol{R}$ ). Let us equip $\mathscr{H}^{x}$ with a scalar product $(\cdot, \cdot)$ defined as

$$
\begin{aligned}
\left(\omega_{\beta_{1}}^{x}, \omega_{\beta_{2}}^{x}\right) & =\left(\omega_{\beta_{1}}^{x}, \omega_{\beta_{2}}^{x}\right)_{\mathscr{T} x}=\left(\beta_{1}, \beta_{2}\right)_{\tilde{\mathcal{H}}}=\int_{0}^{t} \beta_{1}(\tau) \cdot \beta_{2}(\tau) d \tau \\
& =\int_{0}^{t} \frac{d \omega_{\beta_{1}}^{x}(\tau)}{d \tau} \frac{d \omega_{\beta_{2}}^{x}(\tau)}{d \tau} d \tau
\end{aligned}
$$

where $\beta_{1}(\tau), \beta_{2}(\tau), \frac{d \omega_{\beta_{1}}^{x}(\tau)}{d \tau}$ and $\frac{d \omega_{\beta_{2}}^{x}(\tau)}{d \tau}$ are all vectors in $\boldsymbol{R}^{d}$. Then $\mathscr{H}^{x}$ becomes also a separable Hilbert space (isometrically isomorphic with $\widetilde{\mathscr{H}}$ ). Let us denote by $g^{x}$ the (2.3) isomorphy mapping $\widetilde{\mathscr{H}}$ to $\mathscr{H}^{x}$, i. e.

$$
\mathfrak{g}^{x}: \widetilde{\mathscr{H}} \longrightarrow \mathscr{H}^{x} ; \mathfrak{I}^{x}(\beta)=\omega_{\beta}^{x}
$$

and by $S^{x}$ the $g^{x}$ image of $\tilde{S}$, i.e.

$$
S^{x}=g^{x}(\tilde{S}) .
$$

In particular, every element $s^{x} \in S^{x}$ can be expressed as the value of map $g^{x}$ at some point $\tilde{s} \in \tilde{S}$. Thus from (2.1) and (2.3) we have that every $s^{x} \in S^{x}$ has the polygonal form

$$
\begin{aligned}
S^{x}(\tau)=\left[g^{x}(\tilde{S})\right](\tau) & =\sum_{i=0}^{n} \alpha_{i} \cdot\left(-\int_{\tau}^{t} g_{t_{\imath}}\left(\tau^{\prime}\right) d \tau^{\prime}\right)+x \\
& =\sum_{i=0}^{n} \alpha_{\imath} \cdot G_{t_{\imath}}(\tau)+x
\end{aligned}
$$

where $G_{t_{2}}(\tau)=-\int_{\tau}^{t} g_{t_{2}}\left(\tau^{\prime}\right) d \tau^{\prime}=t-\tau \vee t_{2}$ and $\tau \vee t_{2}=\max \left(\tau, t_{2}\right)$. The subset of $S^{x}$ which consists of all polygonal paths (2.8) given by the sum of exactly $n+1$ terms $(i=0,1, \cdots, n)$ we denote by $S_{n}^{x}$. Thus $S^{x}=\bigcup_{n=0}^{\infty} S_{n}^{x}$. From the abstract point of view $S_{n}^{x}$ may be identified with

where

$$
\begin{aligned}
& \Omega_{n}^{x}=\left(x, \boldsymbol{R}^{d} \times(\overbrace{\left.\boldsymbol{R}^{d} \backslash\{0\}\right) \times \cdots \times\left(\boldsymbol{R}^{d} \backslash\{0\}\right)}^{n}, \Delta_{\llcorner 0, t \jmath}^{n})\right. \\
& \Omega_{0}^{x}=\left(x, \boldsymbol{R}^{d}, 0\right)
\end{aligned}
$$

$$
\begin{aligned}
& \Delta_{[0, t]}^{n}=\left\{\left(t_{0}, t_{1}, \cdots, t_{n}\right): t_{0}=0,0<t_{1}<t_{2}<\cdots<t_{n}<t\right\} \\
& \Delta_{[0, t]}^{0}=\{0\} .
\end{aligned}
$$

As the consequence of the identification of $S_{n}^{x}$ and $\Omega_{n}^{x}$ we have that $\Omega^{x}=$ $\bigcup_{n=0}^{\infty} \Omega_{n}^{x}$ is identical with $S^{x}$ 。 


\section{Step 2. The Measure on Polygonal Paths}

The Borel structure on every $\Omega_{n}^{x}, n \in N$, is assumed to be the standard one for this subset of $\boldsymbol{R}^{d(2 n+1)}$ Euclidean space [DSch]. The Borel structure on $S_{n}^{x}$ is induced from the Borel structure on $\Omega_{n}^{x}$ by the isomorphic mapping $\mathcal{g}^{x}$. Let us denote a Borel $\sigma$-algebra in $\Omega_{n}^{x}$ by $\mathcal{B}_{n}^{x}$ and its representation in $S_{n}^{x}$ by $\mathcal{S}_{n}^{x}$. On measurable spaces $\left(\Omega_{n}^{x}, \mathscr{B}_{n}^{x}\right)$ (and $\left(S_{n}^{x}, \mathcal{S}_{n}^{x}\right)$ ) we define a complex (bounded) measure $\mu_{n}$ by setting

$$
\mu_{n}\left(A_{n}\right)=\mu_{n}\left(B_{n}\right)=\int \ldots \int(-i)^{n} \exp \left[i\left(\sum_{l=0}^{n} \alpha_{l}\right) x\right] d \nu\left(\alpha_{0}\right) d \bar{\mu}\left(\alpha_{1}\right) \cdots d \bar{\mu}\left(\alpha_{n}\right) d t_{1} \cdots d t_{n}
$$

where $B_{n} \in \mathcal{B}_{n}^{x}, A_{n}=\mathcal{I}^{x}\left(B_{n}\right) \in \mathcal{S}_{n}^{x}, \nu$ and $\bar{\mu}$ are (bounded) complex measures on $\boldsymbol{R}^{d}$ [DSch, Rud, OPC]. On the space $\Omega^{x}$ (and thus also on $S^{x}$ ), we define measure $\mu$ as follows. As all $\Omega_{n}^{x}$ are treated as mutually disjoint in $\Omega^{x}$ we can define in $\Omega^{x}=\bigcup_{n=0}^{\infty} \Omega_{n}^{x}$ a $\sigma$-algebra $\mathcal{B}^{x}$ as a collection of subsets $B$ of $\Omega^{x}$ which satisfy

$$
\underset{n \in N}{\forall} B \cap \Omega_{n}^{x} \in \mathscr{B}_{n}^{x}
$$

(where $\mathscr{B}_{n}^{x}$ is a Borel $\sigma$-algebra in $\Omega_{n}^{x}$ ). A measure $\mu$ on $\mathscr{B}^{x}$ we then define by

$$
\mu(B)=\sum_{n=0}^{\infty} \mu_{n}\left(B \cap \Omega_{n}^{x}\right)
$$

where $B \in \mathcal{B}^{x}$ and $B \cap \Omega_{n}^{x} \in \mathscr{B}_{n}^{x}$. It is rather easy to check that $\mathcal{B}^{x}$ is well defined $\sigma$-algebra, that the series on the RHS of (2.13) is convergent and that $\mu$ is a well defined measure on $\left(\Omega^{x}, \mathscr{B}^{x}\right)$ [Roy, Ch. 11]. The identical $\sigma$ algebra, denoted $\mathcal{S}^{x}$, and measure $\mu$ are induced in $S^{x}=\bigcup_{n=0}^{\infty} S_{n}^{x}$ by $\mathcal{g}^{x}$.

\section{Step 3. The Measure on the Space $\mathfrak{A}^{x}$ of Alsolutely Continuous Trajectories}

From the above construction we have a measure space $\left(S^{x}, \mathcal{S}^{x}, \mu\right)$. Let us denote by $\mathscr{T}^{x}$ the (Borel) $\sigma$-algebra in $\mathscr{H}^{x}$ generated by topology induced by scalar product (2.5) in this space. Let $\mathscr{H}_{0}$ be

$$
\mathscr{H}_{0}=\mathscr{H}^{x} \backslash \mathcal{S}^{x}
$$

and let us consider the following family $\mathscr{I}_{0}$ of subsets $A$ of $\mathscr{H}_{0}$ : we say that $A \in \mathscr{F}_{0}$ iff there exists $U \in \mathscr{F}^{x}$ such that $A=U \backslash S^{x}$. We can easily check that

(i) $\mathscr{H}_{0} \in \mathscr{F}_{0}$

(ii) $A^{c} \in \mathscr{F}_{0}$ if $A \in \mathscr{F}_{0} \quad\left(A^{c}\right.$ is a complement of $A$ in $\mathscr{H}_{0}$ )

(iii) $\bigcup_{k=0}^{\infty} A_{k} \in \mathscr{I}_{0}$ if $\underset{k=0,1, \cdots}{\forall} A_{k} \in \mathscr{F}_{0}$

i. e. that $\mathscr{I}_{0}$ is a $\sigma$-algebra. 
Let us denote by $\mu_{0}$ a (trivial) zero measure on a measurable space $\left(\mathscr{H}_{0}, \mathscr{F}_{0}\right)$ i. e., we have

$$
\mu_{0}(A)=0 \quad \text { for any } A \in \mathscr{F}_{0} .
$$

Now, we can (trivially) extend measure $\mu$ from $S^{x}$ to the whole space $\mathscr{H}^{x}$ (let us denote the extended measure also by $\mu$ ) by setting

$$
\mu(U)=\mu\left(U \cap S^{x}\right)+\mu_{0}\left(U \cap \mathcal{A}_{0}\right)=\mu\left(U \cap S^{x}\right)
$$

for any $U \in \mathscr{F}^{x}$. As an obvious consequence of (2.17) we have

and

$$
\mu(A)=0 \quad \text { for any } \quad A \in \mathscr{F}_{0}
$$

$$
\mu\left(\mathscr{H}^{x}\right)=\mu\left(S^{x}\right) .
$$

Thus on the path space $\mathscr{H}^{x}$ we have constructed a unique measure $\mu$ which is concentrated on the subset $S^{x}$ of polygonal paths.

\section{Step 4. The Integral with Respect to Measure \&}

Let us consider the functional integral $\int_{\mathscr{r} x} \exp \left[-(i / 2 m)\left(\omega^{x}, \omega^{x}\right)\right] d \mu\left(\omega^{x}\right)$ over the path space $\mathscr{H}^{x}$. Thanks to the definition of measure $\mu$ given by (2.11), (2.13) and (2.17) and properties of function $\exp \left[-(i / 2 m)\left(\omega^{x}, \omega^{x}\right)\right]$ this integral is well defined for all $m \in C_{+}$. Moreover, we can write

$$
\begin{aligned}
\int_{\mathscr{H} x} \exp \left[-\frac{i}{2 m}\left(\omega^{x}, \omega^{x}\right)\right] d \mu\left(\omega^{x}\right) & =\int_{S^{x}} \exp \left[-\frac{i}{2 m}\left(s^{x}, s^{x}\right)\right] d \mu\left(s^{x}\right) \\
& =\sum_{n=0}^{\infty} \int_{S_{n}^{x}} \exp \left[-\frac{i}{2 m}\left(s_{n}^{x}, s_{n}^{x}\right)\right] d \mu_{n}\left(s_{n}^{x}\right)
\end{aligned}
$$

where $s_{n}^{x}$ has a form (2.8) with $n$ fixed. If, moreover, we use the property (2.8a) in the evaluation of the scalar product $\left(s_{n}^{x}, s_{n}^{x}\right)$, take into account the identity

$$
\begin{aligned}
& \left(t-t_{n}\right)\left(\alpha_{n}+\cdots+\alpha_{0}\right)^{2}+\cdots+\left(t_{2}-t_{1}\right)\left(\alpha_{1}+\alpha_{0}\right)^{2}+t_{1} \alpha_{0}^{2} \\
& =\sum_{i, j=0}^{n}\left(t-t_{2} \vee t_{j}\right) \alpha_{i} \alpha_{j}
\end{aligned}
$$

and substitute the explicit form (2.11) of measure $\mu_{n}$ we find from (2.19) that

$$
\begin{aligned}
& \int_{\mathfrak{H} x} \exp \left[-\frac{i}{2 m}\left(\omega^{x}, \omega^{x}\right)\right] d \mu\left(\omega^{x}\right) \\
& =\sum_{n=0}^{\infty}(-i)^{n} \int_{\Omega_{n}^{x}} \exp \left[-\frac{i}{2 m} \sum_{k=0}^{n}\left(\sum_{l=0}^{k} \alpha_{l}\right)^{2}\left(t_{k+1}-t_{k}\right)\right] \\
& \quad \times \exp \left[i\left(\sum_{i=0}^{n} \alpha_{i}\right) x\right] d \nu\left(\alpha_{0}\right) d \bar{\mu}\left(\alpha_{1}\right) \cdots d \bar{\mu}\left(\alpha_{n}\right) d t_{1} \cdots d t_{n} 。
\end{aligned}
$$




\section{Step 5. The Integral on $\mathscr{H}^{x}$ and the Wave Function}

Let us assume that the potential $\mathcal{V}$ and initial condition $\psi_{0}$ for a nonrelativistic quantum system determined by Hamiltonian $H\left(x, \nabla_{x}\right)=-(1 / 2 m) \Delta_{x}+C \mathcal{V}^{\prime}(x)$ both belong to class $\nu^{1}$, and let us choose in particular, that

$$
\mu_{\mathrm{v}}=\bar{\mu} \quad \text { i. e. } \quad \mathcal{Q}(x)=\int_{R^{d}} e^{i a \cdot x} d \mu_{\mathrm{v}}(\alpha)=\int_{R^{d}} e^{i a \cdot x} d \bar{\mu}(\alpha)
$$

and

$$
\phi_{0}(x)=\int_{\boldsymbol{R}^{d}} e^{i a_{0} \cdot x} d \nu\left(\alpha_{0}\right), \quad x \in \boldsymbol{R}^{d} .
$$

Then we can express the $\mathcal{L}^{2}$ solution, $\phi \in \mathcal{L}^{2}\left(\boldsymbol{R}^{d}\right)$, of the Cauchy problem for Schrödinger equation by the Dyson series. In this case, i.e. when $\phi_{0}$ and $\mathscr{V}$ both belong to $\nu^{1}, \psi(t)$ is given by the series which is convergent not only in $\mathcal{L}^{2}\left(\boldsymbol{R}^{d}\right)$ sense but also pointwise, namely

$$
\begin{aligned}
\psi(x, t)= & \sum_{n=0}^{\infty} D_{n}(x, t)=\sum_{n=0}^{\infty}(-i)^{n} \int_{\Delta_{[0, t]}^{n}} d t_{1} \cdots d t_{n} \\
& \times\left[e^{-i\left(t-t_{n}\right) I I_{0}} V e^{-i\left(t_{n}-t_{n-1}\right)^{H_{0}} \cdots V} \mathrm{Ve}^{-i t_{1} H_{0}}\right] \psi_{0}(x) \\
= & \sum_{n=0}^{\infty}(-i)^{n} \int_{\Omega_{n}^{x}} \exp \left[-\frac{i}{2 m}\left(\sum_{l=0}^{k} \alpha_{l}\right)^{2}\left(t_{k+1}-t_{k}\right)\right] \\
& \times \exp \left[i\left(\sum_{i=0}^{n} \alpha_{i}\right) x\right] d \nu\left(\alpha_{0}\right) d \mu_{v}\left(\alpha_{1}\right) \cdots d \mu_{v}\left(\alpha_{n}\right) d t_{1} \cdots d t_{n}, \\
& x \in \boldsymbol{R}^{d}, t \in(0, \infty)
\end{aligned}
$$

where $H_{0}=-(1 / 2 m) \Delta_{x}\left(\Delta_{x}\right.$ denotes Laplacian in $\left.\boldsymbol{R}^{d}\right)$ is a free part of the Hamiltonian $H=H_{0}+V$ ( $V$ is the multiplicative operator in $\mathcal{L}^{2}\left(\boldsymbol{R}^{d}\right)$ induced by $\left.\mu_{v} \in \nu^{1}\right)$. The identity of expressions (2.21) and (2.23) means that the wave function $\psi(t)$, in its pointwise representation (2.23), and the parameterized by $x$ and $t$ functional integral $\int_{\mathscr{H} x} \exp \left[-(i / 2 m)\left(\boldsymbol{\omega}^{x}, \boldsymbol{\omega}^{x}\right)\right] d \mu\left(\boldsymbol{\omega}^{x}\right)$ have equal values for every $x \in \boldsymbol{R}^{d}$ and $t \in(0, \infty)$. In particular it means that $\int_{\mathscr{H} x} \exp \left[-(i / 2 m)\left(\omega^{x}, \omega^{x}\right)\right] d \mu\left(\omega^{x}\right)$ is the pointwise $\mathcal{L}^{2}\left(\boldsymbol{R}^{d}\right)$ solution of the Schrödinger equation with Hamiltonian $H$ on time interval $(0, \infty)$. Let us summarize the above results in the form of

Theorem 1. Let $m \in C_{+}, t \in(0, \infty), \psi_{0}$ and $\mathcal{V} \in \mathcal{\nu}^{1}$ and $a \equiv 0$. Then there exists a measure $\mu$ on the Hilbert space $\mathscr{M}^{x}$, which is concentrated on polygonal paths $S^{x}$, such that the Schrödinger evolution $\phi \in \mathrm{SEV}\left(\nu^{1} ; \nu^{1} ; a \equiv 0\right)$ is represented at any point $x \in \boldsymbol{R}^{d}$ and for any $t \in(0, \infty)$ by the functional integral $\int_{\mathscr{I} x} \exp \left[-(i / 2 m)\left(\omega^{x}, \omega^{x}\right)\right] d \mu\left(\omega^{x}\right)$ where $\omega^{x}(t)=x$.

The above result is crucial for few equivalent formulations of Feynman path integrals in nonrelativistic quantum mechanics in the absence of electromagnetic field [AHK, MC, Tr, CSt]. The real difference between these 
formulations stems from different interpretations of the functional integral $\int_{\mathscr{H} x} \exp \left[-(i / 2 m)\left(\omega^{x}, \omega^{x}\right)\right] d \mu\left(\omega^{x}\right)$ as the Feynman path itnegral which we discuss below.

\section{The Interpretation}

(i) Maslov-Čebotariev interpretation $[\mathrm{MC}]$.

First let us observe that every $\alpha_{i}$ which appears in polygonal trajectories of the form (2.8) can be interpreted as a change in momentum (or velocity) at an instant $t_{i}(i=0,1,2, \cdots)$. This allows us to interpret $\mu_{n}$ as a measure on polygonal trajectories representing sequences of velocity alterations. These velocity changes are caused by instantaneous, independent interactions and are characterized by complex valued distributions $\mu_{v}$ (and $\nu$ at the initial moment). Between instantaneous interactions, the evolution is free so the action functional on the $s_{n}^{x}$ polygonal path can be expressed as the sum of free actions over all straight segments of this trajectory, i. e. it is $1 / 2 m\left(s_{n}^{x}, s_{n}^{x}\right)$. Thus the momentum representation of the amplitude of probability on polygonal trajectories may be written as $\exp \left[-(i / 2 m)\left(s_{n}^{x}, s_{n}^{x}\right)\right]$. Consequently, $\int_{S_{n}^{x}} \exp \left[-(i / 2 m)\left(s_{n}^{x}, s_{n}^{x}\right)\right] d \mu_{n}\left(s_{n}^{x}\right)$ is a weighted (with respect to $n$-interaction sequences) sum of free probability amplitude over $S_{n}^{x}$ (the set of $n$-vertex polygonal trajectories). Therefore $\int_{\mathscr{H} x} \exp \left[-(i / 2 m)\left(\omega^{x}, \omega^{x}\right)\right] d \mu\left(\omega^{x}\right)=\sum_{n=0}^{\infty} \int_{S_{n}^{x}} \exp \left[-(i / 2 m)\left(s_{n}^{x}, s_{n}^{x}\right)\right] d \mu_{n}\left(s_{n}^{x}\right)$ can be recognized as "a sum over all trajectories, of the probability amplitude" of the quantum system under consideration.

This interpretation is in fact better understood in the context of $p$-representation of Schrödinger equation.

For such an equation, with initial condition $\phi_{0}$ and potential $\mathscr{V}$ given via measures $\nu$ and $\mu_{v}$ on the space of velocities $\alpha, \alpha \in \boldsymbol{R}^{d}$, the term $\exp \left[i\left(\sum_{i=0}^{n} \alpha_{i}\right) x\right]$ is absent in the Dyson series (2.23). Thus measure (2.11) is also postulated without this term and the above described interpretation becomes more straightforward $[\mathrm{MC}]$.

(ii) Albeverio-Høegh-Krohn interpretation [AHK].

Lemma 1. For the functional $f_{n}$ on $S_{n}^{x}$ defined as

$$
f_{n}\left(s_{n}^{x}\right)=\int_{S_{n}^{x}} \exp \left[i\left(s_{n}^{x}, s_{n}^{\prime x}\right)\right] d \mu_{n}\left(s_{n}^{\prime x}\right)
$$

we have

$$
\int_{s_{n}^{x}} \exp \left[\frac{i m}{2}\left(s_{n}^{x}, s_{n}^{x}\right)\right] f_{n}\left(s_{n}^{x}\right) d s_{n}^{x}=\int_{s_{n}^{x}} \exp \left[-\frac{i}{2 m}\left(s_{n}^{x}, s_{n}^{x}\right)\right] d \mu_{n}\left(s_{n}^{x}\right)
$$


where $\operatorname{Im} m>0$ (for $m \in \boldsymbol{R} \backslash\{0\}$ (2.25) holds in the sense of analytic continuation), $d \mu_{n}$ is defined by (2.11) and $\int_{S_{n}^{x}}(\cdot) d s_{n}^{x}$ is understood as normalized integral taken over all possible values $y_{k} \in \boldsymbol{R}^{d}$ that trajectories $s_{n}^{x}$ assume at moments $t_{k}$, i.e.,

$$
d s_{n}^{x}=\prod_{k=0}^{n}\left(2 \pi i \frac{t_{k+1}-t_{k}}{m}\right)^{-d / 2} d s_{n, 0}^{x} d s_{n, 1}^{x} \cdots d s_{n, n}^{x}
$$

where $s_{n, k}^{x}=s_{n}^{x}\left(t_{k}\right)=y_{k} \in \boldsymbol{R}^{d}, d s_{n, k}^{x}=d y_{k}, k=0, \cdots, n$.

Proof. If we substitute (2.11), (2.20), (2.24) and (2.26) into the LHS of (2.25) and notice that Fubini's Theorem can be applied to the obtained expression we find

$$
\begin{aligned}
& \int_{S_{n}^{x}} \exp \left[\frac{i m}{2}\left(s_{n}^{x}, s_{n}^{x}\right)\right] f_{n}\left(s_{n}^{x}\right) d s_{n}^{x} \\
& =(-i)^{n} \int_{\Omega_{n}^{x}} d t_{1} \cdots d t_{n} d \nu\left(\alpha_{0}^{\prime}\right) d \mu_{v}\left(\alpha_{1}^{\prime}\right) \cdots d \mu_{\nu}\left(\alpha_{n}^{\prime}\right) \\
& \quad \times\left\{\int_{n_{X}+1} \prod_{\boldsymbol{R}^{d}}^{n}\left(2 \pi i \frac{t_{k+1}-t_{k}}{m}\right)^{-d / 2} d y_{0} \cdots d y_{k}\right. \\
& \quad \times \exp \left[\frac{i m}{2} \sum_{k=0}^{n}\left(\sum_{j=0}^{k} \alpha_{\jmath}\right)^{2}\left(t_{k+1}-t_{k}\right)\right] \\
& \left.\quad \times \exp \left[i \sum_{k=0}^{n}\left(\sum_{j=0}^{k} \alpha_{j}\right)\left(\sum_{l=0}^{k} \alpha_{l}^{\prime}\right)\left(t_{k+1}-t_{k}\right)\right]\right\} \exp \left[i x\left(\sum_{k=0}^{n} \alpha_{k}^{\prime}\right)\right]
\end{aligned}
$$

where $s_{n}^{x}$ and $s_{n}^{\prime x}$ are respectively,

$$
s_{n}^{x}(\tau)=\sum_{i=0}^{n} \alpha_{i} \cdot G_{t_{\imath}}(\tau) ; \quad s_{n}^{\prime x}=\sum_{i=0}^{n} \alpha_{i}^{\prime} \cdot G_{t_{\imath}}(\tau) .
$$

Substituting now identities

$$
\left(\sum_{\jmath=0}^{k} \alpha_{\jmath}\right)\left(t_{k+1}-t_{k}\right)=y_{k+1}-y_{k}
$$

and

$$
m\left(\sum_{\jmath=0}^{k} \alpha_{\jmath}\right)^{2}\left(t_{k+1}-t_{k}\right)=m\left(y_{k+1}-y_{k}\right)^{2}\left(t_{k+1}-t_{k}\right)^{-1}
$$

to (2.27) and performing Fresnel type integration wrt $d y_{0} \cdots d y_{k}$ in bracket $\{\cdots\}$ we obtain

$$
\begin{aligned}
(2.27)= & (-i)^{n} \int_{\Omega_{n}^{x}} d t_{1} \cdots d t_{n} d \nu\left(\alpha_{0}^{\prime}\right) d \mu_{v}\left(\alpha_{1}^{\prime}\right) \cdots d \mu_{v}\left(\alpha_{n}^{\prime}\right) \\
& \times \exp \left[-\frac{i}{2 m} \sum_{k=0}^{n}\left(\sum_{l=0}^{k} \alpha_{l}^{\prime}\right)^{2}\left(t_{k+1}-t_{k}\right)\right] \cdot \exp \left[i x\left(\sum_{k=0}^{n} \alpha_{k}^{\prime}\right)\right] \\
= & \int_{S_{n}^{x}} \exp \left[-\frac{i}{2 m}\left(s_{n}^{\prime x}, s_{n}^{\prime x}\right)\right] d \mu_{n}^{x}\left(s_{n}^{\prime x}\right) .
\end{aligned}
$$

For $m \in \boldsymbol{R} \backslash\{0\}$ the equalities (2.27) and (2.28) can be interpreted in the sense of 
analytic continuation.

Let us notice now that $f_{n}\left(s_{n}^{x}\right)$ given by $(2.24)$ and (2.11), i.e.

$$
\begin{aligned}
J_{n}\left(S_{n}^{u}\right)= & \dot{J}_{S_{n}^{x}} \exp \left\lfloor\imath\left(s_{n}^{x}, s_{n}^{\prime}\right)\right] d \mu_{n}\left(s_{n}^{\prime x}\right) \\
= & (-i)^{n} \int_{\Omega_{\frac{x}{u}}} d \nu\left(\alpha_{0}^{\prime}\right) d \mu_{0}\left(\alpha_{1}^{\prime}\right) \cdots d \mu_{0}\left(\alpha_{n}^{\prime}\right) d t_{1} \cdots d t_{n} \\
& \times \exp \left[i \sum_{k=0}^{n}\left(\sum_{j=0}^{k} \alpha_{\jmath}\right)\left(\sum_{l=0}^{k} \alpha_{l}^{\prime}\right)\left(t_{k+1}-t_{k}\right)\right] \exp \left[i x\left(\sum_{k=0}^{n} \alpha_{k}^{\prime}\right)\right] \\
= & (-i)^{n} \int_{\Omega_{n}^{x}} d \nu\left(\alpha_{0}^{\prime}\right) d \mu_{0}\left(\alpha_{1}^{\prime}\right) \cdots d t_{n} \exp \left[i \sum_{k, l=0}^{n} \alpha_{l} \alpha_{k}^{\prime}\left(t-t_{l} \vee t_{k}\right)\right] \\
& \times \exp \left[i x\left(\sum_{k=0}^{n} \alpha_{k}^{\prime}\right)\right]
\end{aligned}
$$

can be written, thanks to the form $(2.22 \mathrm{a}, \mathrm{b})$ of $\phi_{0}$ and $\checkmark$, as

$$
\begin{aligned}
& f_{n}\left(s_{n}^{x}\right)=(-i)^{n} \int_{\Omega_{n}^{x}} d \nu\left(\alpha_{0}^{\prime}\right) d \mu_{v}\left(\alpha_{1}^{\prime}\right) \cdots d t_{n} \\
& \times \exp \left\{i \sum_{k=0}^{n} \alpha_{k}^{\prime}\left[\left(\sum_{l=0}^{n} \alpha_{l}\right)\left(t-t_{l} \vee t_{k}\right)+x\right]\right\} \\
& =(-i)^{n} \int_{\Omega_{n}^{x}} d \nu\left(\alpha_{0}^{\prime}\right) d \mu_{\nu}\left(\alpha_{1}^{\prime}\right) \cdots d t_{n} e^{i s_{n}^{x}\left(t_{0}\right) a_{0}^{\prime}} \\
& \times e^{i s s_{n}^{x}\left(t_{1}\right) a_{1}^{\prime}} \ldots e^{i s_{n}^{x}\left(t_{n}\right) a_{n}^{\prime}} \\
& =\frac{(-i)^{n}}{n !}\left(\int_{0}^{t} \mathcal{} \mathcal{}\left(s_{n}^{x}(\tau)\right) d \tau\right)^{n} \psi_{0}\left(s_{n}^{r}(0)\right) \text {. }
\end{aligned}
$$

The equality (2.25) suggests that for the functional $f$ on $\mathscr{H}^{x}$ defined as a natural extension of functions $f_{n}$ on $S_{n}^{x}, n \in \mathbb{N}$, i. e.

$$
f\left(\omega^{x}\right)=\int_{\mathscr{K} x} \exp \left[i\left(\omega^{x}, \omega^{\prime x}\right)\right] d \mu\left(\omega^{\prime x}\right)=\sum_{n=0}^{\infty} \int_{S_{n}^{x}} e^{i\left(\omega^{x}, s_{n}^{\prime x}\right)} d \mu_{n}\left(s_{n}^{\prime x}\right)
$$

the equality

$$
\int_{\mathscr{H}} e^{i(m / 2)\left(\omega^{x}, \omega^{x}\right)} f\left(\omega^{x}\right) d \omega^{x}=\int_{\mathscr{T} x} e^{-(i / 2 m)\left(\omega^{x}, \omega^{x}\right)} d \mu\left(\omega^{x}\right)
$$

should be satisfied. However, it is easy to realize that identity (2.32) cannot be proved. In fact, the LHS of (2.32) is meaningless, as it stands, since we have not any natural, Lebesgue-like measure $d \omega^{x}$ on $\mathscr{H}^{x}$. Therefore (2.32) must be seen as the definition of the expression on its LHS for which (2.25) provides more or less satisfactory justification. Let us notice here that (2.25) is the best justification of equality (2.32) which is available. Without the explicit construction of measure $\mu$ even the version (2.25) of this equality, restricted to the dense set $S^{x}$ of $\mathscr{H}^{x}$, is unknown and (2.32) has to be assumed ad hoc [AHK]. 
If we finally combine relations (2.31), (2.30) and (2.32) and recall Theorem 1 we find that the wave function $\phi(\cdot, t)$ of the quantum system determined by $\phi_{0}$ and $C V$ given by $(2.22 \mathrm{a}, \mathrm{b})$ can be expressed at any point $x \in \mathbb{R}^{d}$ and for any $t \in(0, \infty)$ as

$$
\begin{aligned}
\psi(x, i) & =\int_{\mathscr{T} x} \exp \left[-\frac{i}{2 m}\left(\omega^{x}, \omega^{x}\right)\right] d \mu\left(\omega^{x}\right) \\
& =\int_{\mathscr{T} x} \exp \left[i\left(\int_{0}^{t}\left\{\frac{m}{2}\left|\frac{d \omega^{x}(\tau)}{d \tau}\right|^{2}-\mathcal{}-\mathcal{\nu}\left(\omega^{x}(\tau)\right)\right\} d \tau\right)\right] \psi_{0}\left(\omega^{x}(0)\right) d \omega^{x}
\end{aligned}
$$

i.e. that it represents the "sum over all trajectories of the probability amplitude $\exp \left[i\left(S_{0}(x)+S_{I}(x)\right)\right] "$.

Reniark. It is also possible to perform the similar construction in the circumstance when the family of regular polygonal trajectories is chosen [MC, $\mathrm{Tr}$ ] as the dense set in $\mathscr{H}^{x}$ instead of $S^{x}$. By regular polygonal trajectories we understand polygonals with uniformly distributed vertices over the time axis (i. e. for fixed $n$ we have that $t_{2}$ in (2.1) and (2.8) are simply $t_{i}=(i / n+1) t, i=$ $0, \cdots, n+1)$. For such trajectories the spaces $\Omega_{n}^{x}(2.9)$ will collapse to $\left.\Omega_{n}^{x}\right|_{r}=$ $\left(x, \stackrel{n+1}{\times} \boldsymbol{R}^{d}\right)$ and, in an obvious way, measures $\mu_{n}(2.11)$ on $\Omega_{n}^{x}$, measure $\mu(2.13)$, (2.17) on $\mathscr{H}^{x}$ and functional integral $\int_{\mathscr{K} x} \exp \left[-(i / 2 m)\left(\omega^{x}, \omega^{x}\right)\right] d \mu\left(\omega^{x}\right)$ will be simplified to measures $\left.\mu_{n}\right|_{r}$ on $\left.\Omega_{n}^{\dot{x}}\right|_{r}$, measure $\left.\mu\right|_{r}$ on $\mathscr{H}^{x}$ and functional integral $\left.\int_{\mathscr{I} x} \exp \left[-(i / 2 m)\left(\omega^{x}, \omega^{x}\right)\right] d \mu\right|_{r}\left(\omega^{x}\right)$, respectively. It can be shown that $\left.\int_{\mathscr{r} x} \exp \left[-(i / 2 m)\left(\omega^{x}, \omega^{x}\right)\right] d \mu\right|_{r}\left(\omega^{x}\right)$ is also equal to the wave function of the quantum system under consideration $[\mathrm{MC}]$ and that the Maslov-Čebotariev [MC] and Albeverio-Hcegh-Krohn [Tr] interpretations are valid also in the case when as the set sustaining the measure $\mu_{I}$ the collection of regular trajectories is chosen. Finally, let us mention that it is possible to find other specific procedures which allow us to approximate the functional integral $\int_{\mathscr{H} x} \exp \left[-(i / 2 m)\left(\omega^{x}, \omega^{x}\right)\right] d \mu\left(\omega^{x}\right)$ on the set of regular polygonals [Tr, Th. 4] or general polygonals [CSt, Ths. $1 \& 2$ ] without in fact constructing measures $\mu$ on any set of polygonal paths [CSt, Tr].

Let us now generalize our results to the case of quantum systems which are under the influence of the external electromagnetic field $a, a \in \nu_{k / 2}^{d}$. The full Hamiltonian for such a system is given by (1.2) and we have the following result.

Theorem 2. Let $m \in \boldsymbol{C}_{+}, \hat{\psi}_{0} \in C_{u}^{\infty}\left(\boldsymbol{R}^{d}\right), \quad \mathcal{V} \in \boldsymbol{\nu}_{k}^{1}, a \in \nu_{k / 2}^{d}$ and $t<T_{\gamma}$. Then there exists a measure $\lambda$ on the Hilbert space $\mathscr{H}^{x}$, which is concentrated on polygonal paths $S^{x}$, such that the Schrödinger evolution $\phi \in \operatorname{SEv}\left(\check{C}_{0}^{\infty}\left(\boldsymbol{R}^{d}\right) ; \nu_{k}^{1}, \nu_{k / 2}^{d}\right)$ is re- 
presented at any point $x \in \boldsymbol{R}^{d}$ and for any $t \in\left(0, T_{\gamma}\right)$ by thefunctional integral

$$
\int_{\mathscr{T} x} \exp \left[-\frac{i}{2 m}\left(\omega^{x}, \omega^{x}\right)\right] d \lambda\left(\omega^{x}\right) \quad \text { where } \omega^{x}(t)=x \text {. }
$$

Proof. (For some details check [OPC], Proposition 3.) First, construct a measure $\lambda_{n}$ on $S_{n}^{x}$ so that the $n$-th term in the formal Dyson series expansion can bewritten as a pointwise function

$$
\begin{aligned}
{\left[D_{n}(t ; m) \psi_{0}\right](x)=} & \int_{\Delta_{[0, t]}^{n}}(-i)^{n} d t_{1} \cdots d t_{n} \\
& \times\left[e^{-\imath\left(t-t_{n}\right) H_{0}} \bar{V} e^{-i\left(t_{n}-t_{n-1}\right) H_{0}} \cdots \bar{V} e^{-i t_{1} H_{0}}\right] \psi_{0}(x) \\
= & \int_{S_{n}^{x}} \exp \left[-\frac{i}{2 m}\left(s_{n}^{x}, x_{n}^{x}\right)\right] d \lambda_{n}\left(s_{n}^{x}\right), \\
& \quad x \in \boldsymbol{R}^{d}, m \in \boldsymbol{C}_{+}, n \in N
\end{aligned}
$$

where $\bar{V}$ is given by (1.2). The explicit formula for $\lambda_{n}$, in analogy to (2.11) is

$$
\begin{aligned}
\lambda_{n}\left(A_{n}\right)=\lambda\left(B_{n}\right)= & \int_{B_{n}}(-i)^{n} \exp \left[i x \sum_{l=0}^{n} \alpha_{l}\right] \hat{\psi}_{0}\left(\alpha_{0}\right) d \alpha_{0} \prod_{l=1}^{n}\left[d \mu^{\prime}\left(\alpha_{l}\right)\right. \\
& \left.-\frac{1}{m}\left(\alpha_{0}+\sum_{j=1}^{l-1} \alpha_{j}+\frac{1}{2} \alpha_{l}\right) \cdot d \gamma\left(\alpha_{l}\right)\right] d t_{1} \cdots d t_{n} \\
= & \int_{P_{\alpha_{0}\left(B_{n}\right)}} d \alpha_{0} \hat{\psi}_{0}\left(\alpha_{0}\right) \int_{B_{n} \mid \alpha_{0}} d \tilde{\lambda}_{n}
\end{aligned}
$$

where $B_{n} \in \mathscr{B}_{n}^{x}, A_{n}=\mathcal{g}^{x}\left(B_{n}\right) \in \mathcal{S}_{n}^{x},\left.B_{n}\right|_{\alpha_{0}}$ denotes the $\alpha_{0}$-section of $B_{n}$ and $P_{\alpha_{0}}\left(B_{n}\right)$ denotes the projection of the set $B_{n}$ in $\boldsymbol{R}^{d(2 n+1)}$ Euclidean space on the $\alpha_{0}$ axis.

The measure $\lambda$ on $S^{x}$ and $\mathscr{H}^{x}$ is then constructed exactly as in the case $a=0$ (Steps $2 \& 3$ of the proof of Theorem 1) but it is a bit more work now to check that the series on the RHS of equality

$$
\lambda(B)=\sum_{n=1}^{\infty} \lambda_{n}\left(B \cap \Omega_{n}^{x}\right)
$$

is convergent. Taking into account that $\hat{\phi}_{0}$ and $\gamma$ both have compact support we readily find from the definition (2.36) of the measure $\lambda_{n}$ that its total variation $\left\|\lambda_{n}\right\|$ can be bounded as follows

$$
\begin{aligned}
\left\|\lambda_{n}\right\|= & \left|\lambda_{n}\right|\left(\boldsymbol{R}^{(2 n+1) d}\right) \leqq\left\|\hat{\psi}_{0}\right\|_{\infty} \operatorname{Vol}\left(\operatorname{supp} \hat{\psi}_{0}\right) \int_{\boldsymbol{R}^{2 n d}} \sum_{l=1}^{n}\left[d\left|\mu^{\prime}\right|\left(\alpha_{l}\right)\right. \\
& \left.+\frac{1}{|m|}\left(b+(l-1) k+\frac{1}{2} k\right) d|\gamma|\left(\alpha_{l}\right)\right] d t_{1} \cdots d t_{n} \leqq\left\|\hat{\psi}_{0}\right\|_{\infty} \operatorname{Vol}\left(\operatorname{supp} \hat{\psi}_{0}\right) \\
{\left[\left\|\mu^{\prime}\right\|+\right.} & \left.\frac{1}{|m|}(b+n k)\|\gamma\|\right]^{n} \frac{t^{n}}{n !} \leqq\left\|\hat{\psi}_{0}\right\|_{\infty} \operatorname{Vol}\left(\operatorname{supp} \hat{\psi}_{0}\right) \frac{t^{n}}{n !}\left[\frac{2 n k\|\gamma\|}{|m|}\right]^{n}=d_{n}
\end{aligned}
$$

for $n$ sufficiently large, where $\left|\mu^{\prime}\right|,|\gamma|,\left|\lambda_{n}\right|$ are total variation measures associated with complex measures $\mu^{\prime}, \gamma$ and $\lambda_{n}$, respectively, and $b$ is the radius 
of the sphere which contains the support of $\hat{\psi}_{0}$. As we have

$$
\lim _{n \rightarrow \infty} \frac{d_{n+1}}{d_{n}}=\frac{2 e k\|\gamma\|}{|m|} t
$$

we can conclude that

$$
\sum_{n=0}^{\infty}\left\|\lambda_{n}\right\|<\infty \quad \text { for } \quad t<\frac{|m|}{2 e k\|\gamma\|}
$$

The above property of measures $\lambda_{n}$ guarantees the convergence of the series (2.37). Thus $\lambda$ is a well defined complex measure on $S^{x}$, and after trivial extension (Step 3), also on $\mathscr{H}^{x}$. In this way we find that $\phi(x, t)=$ $\int_{\mathscr{H} x} \exp \left[-1 / 2 m\left(\boldsymbol{\omega}^{x}, \boldsymbol{\omega}^{x}\right)\right] d \lambda\left(\boldsymbol{\omega}^{x}\right)$ is pointwise well defined function for any $x \in \boldsymbol{R}^{d}$ and $t \in\left(0, T_{\gamma}\right)\left(m \in \boldsymbol{C}_{+}\right)$and that it expresses the value of the formal Dyson series for the Cauchy problem of the Schrödinger equation with Hamiltonian (1.2). Since $\bar{V}$ in (1.2) is an unbounded operator we are no longer assured by the trivial abstract theory that this Dyson series, though pointwise convergent, satisfies the Cauchy problem for the Schrödinger equation in $\mathcal{L}^{2}\left(\boldsymbol{R}^{d}\right)$. Nevertheless, it is possible to derive the recurrence relation [OPC, 5.24]

$$
i \frac{\partial}{\partial t} D_{n}(t ; m) \phi_{0}=H_{0}(m) D_{n}(t ; m) \psi_{0}+\bar{V}(m) D_{n-1}(t ; m) \psi_{0}, \quad \phi_{0} \in \check{C}_{0}^{\infty}\left(\boldsymbol{R}^{d}\right)
$$

in $\mathcal{L}^{2}\left(\boldsymbol{R}^{d}\right)\left(D_{-1}=0\right)$; show the boundedness of sums $\sum_{n=0}^{\infty}\left\|H_{0}(m) D_{n}(t ; m) \psi_{0}\right\|$ and $\sum_{n=0}^{\infty}\left\|V(m) D_{n}(t ; m) \psi_{0}\right\|$; and interchangability of $\partial / \partial t, H_{0}(m)$ and $V(m)$ with infinite sums $\sum_{n=0}^{\infty}[\mathrm{OPC}]$. This allows us to conclude that $\phi(x, t)=\sum_{n=0}^{\infty} D_{n}(t ; m) \phi_{0}(x)=$ $\int_{\mathscr{H} x} \exp \left[-i / 2 m\left(\omega^{x}, \omega^{x}\right)\right] d \lambda\left(\omega^{x}\right)$ is in fact the evolution of the kind $\operatorname{SEv}\left(\check{C}_{0}^{\infty}\left(\boldsymbol{R}^{d}\right)\right.$; $\left.\nu_{k}^{1}, \nu_{k / 2}^{d}\right)$.

Discussion. Measures $\mu$ and $\lambda$ on $\mathscr{T}^{x}$ are both constructed from $V=H-H_{0}$, where $H$ denotes the complete Hamiltonian of evoluations $\operatorname{SEv}\left(\nu^{1} ; \nu^{1} ; a \equiv 0\right)$ and $\operatorname{SEv}\left(\check{C}_{0}^{\infty}\left(\boldsymbol{R}^{d}\right), \nu_{k}^{1}, \nu_{k / 2}^{d}\right)$, respectively, and $H_{0}$ is a free Hamiltonian. This construction guarantees that functional integrals $\int_{\mathscr{H} x} \exp \left[-(i / 2 m)\left(\omega^{x}, \omega^{x}\right)\right] d \mu\left(\omega^{x}\right)$ and $\int_{\mathscr{H} x} \exp \left[-(i / 2 m)\left(\omega^{x}, \omega^{x}\right)\right] d \lambda\left(\omega^{x}\right)$ are equal to the Dyson series representations of solutions of evolution problems $\operatorname{SEv}\left(\nu^{1} ; \nu^{1} ; a \equiv 0\right)$ and $\operatorname{SEv}\left(\check{C}_{0}^{\infty}\left(\boldsymbol{R}^{d}\right), \nu_{k}^{1} ; \nu_{k / 2}^{d}\right)$, respectively. Both these functional integrals express the weighted sum of free probability amplitudes over all absolutely continuous trajectories. They may be seen as the realization of Feynman's idea of path integration in the spirit of Cebotoriev-Maslov. Notice, however, that for $\operatorname{SEv}\left(\nu^{1}, \nu^{1}, a \equiv 0\right)$ the potential $V(x)=H\left(x, \nabla_{x}\right)-H_{0}$ from which $\mu$ is constructed is at the same time the (negative) perturbation in the formula for the complete Lagrangian $L_{\text {com }}=L_{\text {free }}-V(x)$ while for $\operatorname{SEv}\left(\check{C}_{0}^{\infty}\left(\boldsymbol{R}^{d}\right) ; \nu_{k}^{1} ; \nu_{k / 2}^{d}\right)$ this is no longer true. The explicit form of 
$\bar{V}\left(x, \nabla_{x}\right)=H\left(x, \nabla_{x}\right)-H_{0}(1.2)$ from which $\lambda$ is constructed is inconsistent with the perturbation to the free Lagrangian $L_{\text {free }}$ and does not allow us to generalize the Albeverio-Høegh-Krohn interpretation to the case of quantum system under the influence of the external electromagnetic field. Though Lemma 1 can still be proved for the evolution $\operatorname{SEv}\left(\check{C}_{0}^{\infty}\left(\boldsymbol{R}^{d}\right) ; \nu_{k}^{1}, \nu_{k / 2}^{d}\right)$, the (2.30) type equality cannot as the functional $f_{n}$ given by $f_{n}\left(s_{n}^{x}\right)=\int_{S_{n}^{x}} \exp \left[i\left(s_{n}^{x}, s_{n}^{\prime x}\right)\right] d \lambda\left(s_{n}^{\prime x}\right)$ is inconsistent with $\frac{(-i)^{n}}{n !}\left[\int_{0}^{t}\left(\frac{d s_{n}^{x}}{d \tau} \cdot\left(a s_{n}^{x}\right)-\mathcal{V}\left(s_{n}^{x}\right)\right) d \tau\right]^{n} \psi_{0}\left(s_{n}^{x}(0)\right)$. Consequently, the validity of the (2.33) type equality on which the Albeverio-Hoegh-Krohn interpretation is based is unfounded for the quantum evolution in the presence of an external electromagnetic field.

\section{Acknowledgements}

The author gratefully acknowledges helpful discussions with T. Osborn, A. Saksena, R. Corns and F. Molzahn.

\section{References}

[AHK] Albeverio, S. and Høegh-Krohn, R., Mathematical Theory of Feynman Path Integral, (Springer, Berlin, 1976).

[CHRS] Combe, P., Høegh-Krohn, R., Rodriguez, R., Sirugue, M. and Sirugue-Collin, M., Commun. Math. Phys., 77 (1980), 269.

[CM] Cebotariev, A. and Maslov, V., In: Feynman Path Integrals, Lecture Notes in Physics, 106 (1979), 58.

[CSt] Cameron, R. and Storvick, D., Proc. London Math. Soc., 52 (1986), 557.

[DSch] Dunford, N. and Schwartz, J., Linear Operators Part I, (Interscience, New York, 1967).

[MC] Maslov, V. and Cebotariev, A., Soviet Math. Dokl., 17 (1976), 975.

[OPC] Osborn, T., Papiez, L. and Corns, R., J. Math. Phys., 28 (1987), 103.

[Roy] Royden, M., Real Analysis, (The Macmillan Company, New York, 1963)

[Rud] Rudin, W., Real and Complex Analysis, (McGraw-Hill, New York, 1974).

[Tr] Truman, A., J. Math. Phys., 18 (1977), 1499.

Note added at proof: In fact, when deriving (2.33) we combine with (2.32) rather the following generalization (2.30a) of the formula (2.30) i.e.

$$
\begin{aligned}
f\left(\omega^{x}\right) & =\sum_{n=0}^{\infty} f_{n}\left(\omega^{x}\right)=\sum_{n=0}^{\infty} \frac{(-i)^{n}}{n !}\left[\int_{0}^{t} \mathcal{} \mathcal{V}\left(\omega^{x}(\tau)\right) d \tau\right]^{n} \psi_{0}\left(\omega^{x}(0)\right) \\
& =\exp \left[-\int_{0}^{t} \mathcal{L}\left(\omega^{x}(\tau)\right) d \tau\right] \psi_{0}\left(\omega^{x}(0)\right)
\end{aligned}
$$

than the formula (2.30) itself. (2.30a) is a result of the substitution of (2.27a) into the scalar product $\left(\omega^{x}, s^{\prime x}\right)$ and the explicit expansion of $\left(\omega^{x}, s_{n}^{\prime}\right)$ according to (2.5) which shows that

$$
f_{n}\left(\omega^{x}\right)=\int_{S_{n}^{x}} e^{2\left(\omega^{x}, s^{\prime} \frac{x}{n}\right)} d \mu_{n}\left({s^{\prime}}_{n}^{x}\right)=\frac{(-i)^{n}}{n !}\left[\int_{0}^{t} \mathcal{V}\left(\omega^{x}(\tau)\right) d \tau\right]^{n} \psi_{0}\left(\omega^{x}(0)\right)
$$

